\title{
Enhanced Thermoelectric and Mechanical Performance in n-type Yb-filled Skutterudites through Aluminum alloying
}

Dandan Qin ${ }^{\mathrm{a}}{ }^{\dagger}$, Bo Cui ${ }^{\mathrm{b}}{ }^{\dagger}$, Jianbo Zhu ${ }^{\mathrm{a}}$, Wenjing Shi ${ }^{\mathrm{a}}$, Haixu Qin ${ }^{\mathrm{a}}$, Fengkai Guo ${ }^{\mathrm{a}}$, Jian $\mathrm{Cao}^{\mathrm{a}}$, Wei Cai ${ }^{\mathrm{b}}$, and Jiehe Suia, ${ }^{*}$

aState Key Laboratory of Advanced Welding and Joining, Harbin Institute of Technology, Harbin 150001, China

${ }^{b}$ National Key Laboratory for Precision Hot Processing of Metals, Harbin Institute of Technology, Harbin 150001, China

$\dagger$ Equal contributors

*To whom correspondence should be addressed.E-mail: suijiehe@hit.edu.cn (Jiehe Sui)

\section{S- I Materials and Methods}

Sample synthesis: Starting materials including Yb ingot (99.9\%, Alfa Aesar), Co piece (99.95\%, Alfa Aesar), Sb ball (99.999\%, Alfa Aesar), and Al piece (99.9\%, Alfa Aesar) were firstly weighted according to stoichiometric $\mathrm{Yb}_{0.3} \mathrm{Co}_{4} \mathrm{Sb}_{12} / \mathrm{xAl}(\mathrm{x}=0,0.2,0.3,0.4)$ and then sealed in the silica tubes under vacuum. All the procedure mentioned above were carried out in a glove box with Ar atmosphere. The sealed tubes were then transmitted to a box furnace and slowly heated to $1423 \mathrm{~K}$ in $20 \mathrm{~h}$, soaked at that temperature for $3 \mathrm{~h}$ and then furnace cooled to room temperature. The reacted ingots were cleaned and placed in the cleaned quartz tubes with a fine nozzle, which were induction-molten and injected on the copper wheel with a rotating speed of $50 \mathrm{~m} \mathrm{~s}^{-1}$ under the high-purity Ar atmosphere. The obtained ribbons were ground into fine powders in the glove box and then were densified by using the hot-pressed method at $1023 \mathrm{~K}$ for $1 \mathrm{~h}$ in a $12.7 \mathrm{~mm}$ diameter graphite die under an axial pressure of $90 \mathrm{MPa}$ under vacuum of $5 \times 10^{-3} \mathrm{~Pa}$.

Microstructure characterization: The phase structure is determined by powder X-ray diffraction (XRD), with data collected on a PANalyticalX'Pert Pro X-ray 
diffractometer using $\mathrm{Cu} K \alpha$ radiation $(\lambda=1.5418 \AA)$. The morphologies and actual composition are investigated using a HELIOS Nanolab 600i scanning electronic microscope (SEM) accompanied with the energy-dispersive X-ray spectroscopy (EDS). The microstructure is investigated using a Talos F200X high resolution transmission electronic microscopy (HRTEM), where the TEM specimen is prepared by conventional methods, including dicing, grinding, and then Ar ion milling.

Properties characterization: The sintered bulk pellets are cut into bars with dimension of $2 \times 2 \times 12 \mathrm{~mm}^{3}$ for the electrical resistivity and Seebeck coefficient measurement on a system (ULVAC ZEM-3) under low-pressure helium atmosphere from $300 \mathrm{~K}$ to 873 $\mathrm{K}$. The thermal conductivity $\kappa$ can be calculated with the relationship $\kappa=D C_{p} \rho$, where $D$ is thermal diffusivity coefficient, $C_{p}$ is specific capacity and $\rho$ is the density. Disks with diameter of $12.7 \mathrm{~mm}$ and thickness of $1.5 \mathrm{~mm}$ are prepared to measure the thermal diffusivity coefficient $D$ using laser flash analysis method (Netzsch LFA 427) under a continuous nitrogen flow in the temperature range $300 \mathrm{~K}$ to $873 \mathrm{~K}$. The specific capacity $C_{p}$ is measured by DSC system (Netzsch DSC 404). The density is determined by Archimedes method, and relative densities of all samples are higher than $96 \%$. The Hall coefficient $\left(R_{H}\right)$ is measured using the van der Pauw technique under a reversible magnetic field of $1.5 \mathrm{~T}$. The carrier concentration $\left(n_{H}\right)$ and the carrier mobility $\left(\mu_{H}\right)$ are calculated by $n_{H}=1 / \mathrm{e} R_{H}$ and $\mu_{H}=\sigma R_{H}$, respectively. The uncertainty is $3 \%$ for the electrical conductivity, $5 \%$ for the Seebeck coefficient, $7 \%$ for the thermal conductivity, so the combined uncertainty for the power factor and $Z T$ value is $13 \%$ and $20 \%$, respectively. Indentation fracture toughness analysis is carried out using Vickers' microhardness tester (HVS-1000). A load of $0.5 \mathrm{~N}$ and a loading time of $10 \mathrm{~s}$ are used. Nanoindentation experiment is carried out with the Berkovich diamond indenter B-L72. The ultraviolet photoemission spectroscopy (UPS) measurement is performed on a ThermoFisher ESCALAB 250Xi instrument with an He I radiation source $(h v=21.2$ $\mathrm{eV})$. 


\section{S- II Work function calculation:}

The UPS spectrum can highlight the cut-off $E_{\text {cut-off }}$ ( I ) and Fermi edge $E_{\mathrm{F}}$ ( II) (Figure 5). The elastic cut-off position (' I' circle) and the Fermi edge position (' II' circle) were determined using a linear extrapolation method. The spectrum width $(L)$ of the UPS spectrum obtained by subtracting $E_{\text {cut-off }}$ from $E_{\mathrm{F}}\left(L=E_{\mathrm{F}}-E_{\text {cut-off }}{ }^{[1]}\right)$ for the CoAl and matrix are $17.51 \mathrm{eV}$ and $17.1 \mathrm{eV}$, respectively. Therefore, the work function $(W=h v$ $-L$ ) for the CoAl and matrix are about $3.69 \mathrm{eV}$ and $4.1 \mathrm{eV}$, respectively. The $h v$ is the phonon energy and equal to about $21.2 \mathrm{eV}$. The work function of CoAl nanoprecipitates is calculated using the equation for spherical metal particles ${ }^{[2]}$ :

$$
W_{\mathrm{m}}(R)=W_{\infty}+\frac{5.4}{R}
$$

where $W_{\infty}$ is the work function of the bulk metals and $R$ is the radius of the particles (in $\AA) . W_{\infty}(3.69 \mathrm{eV})$ for the bulk CoAl is obtained from the UPS spectrum and $R$ for CoAl nanoprecipitates is about $100-200 \mathrm{~nm}$. Therefore, the $W_{\mathrm{m}}$ of CoAl nanoprecipitates is about $3.69 \sim 3.7 \mathrm{eV}$.

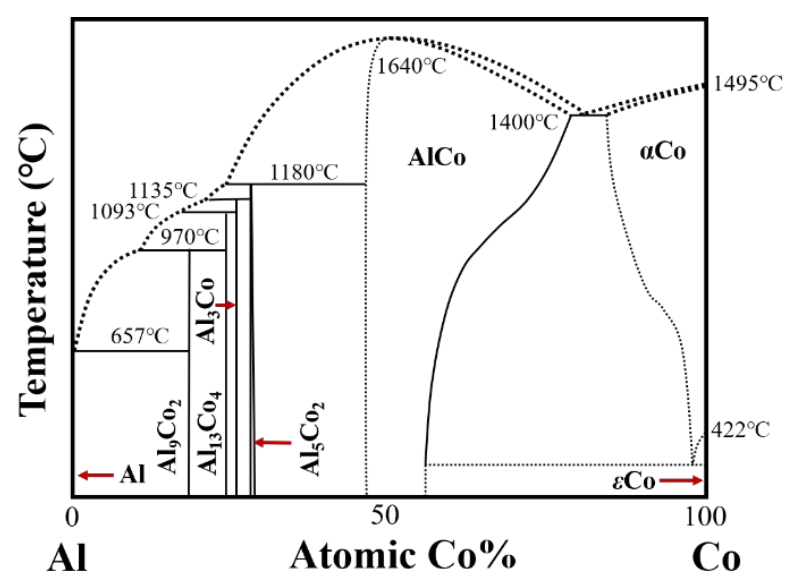

Figure S1 The phase diagram of Co-Al. 


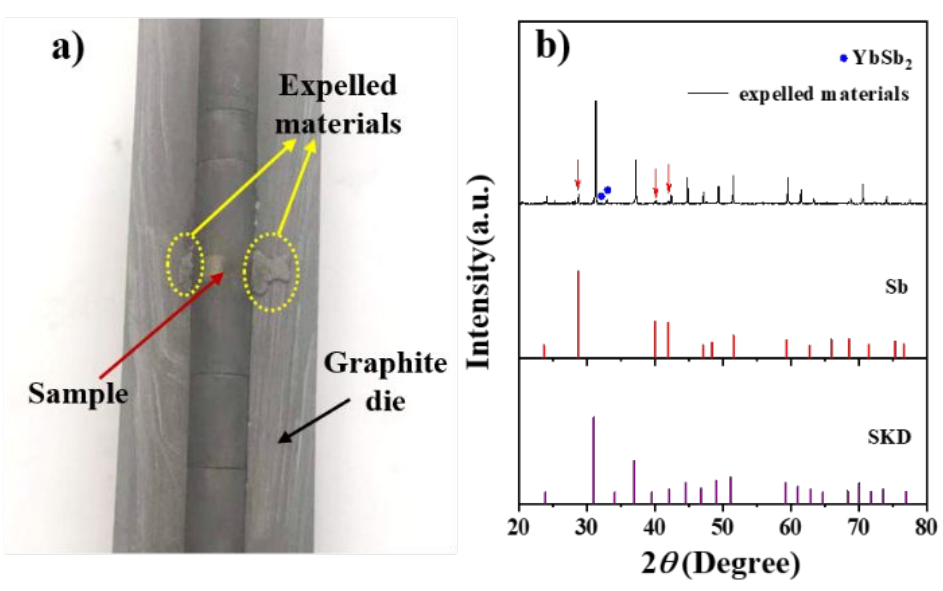

Figure S2 a) Picture after hot press sintering for $\mathrm{Yb}_{0.3} \mathrm{Co}_{4} \mathrm{Sb}_{12} / 0.3 \mathrm{Al}$ sample. b) PXRD patterns of the expelled materials in (a).

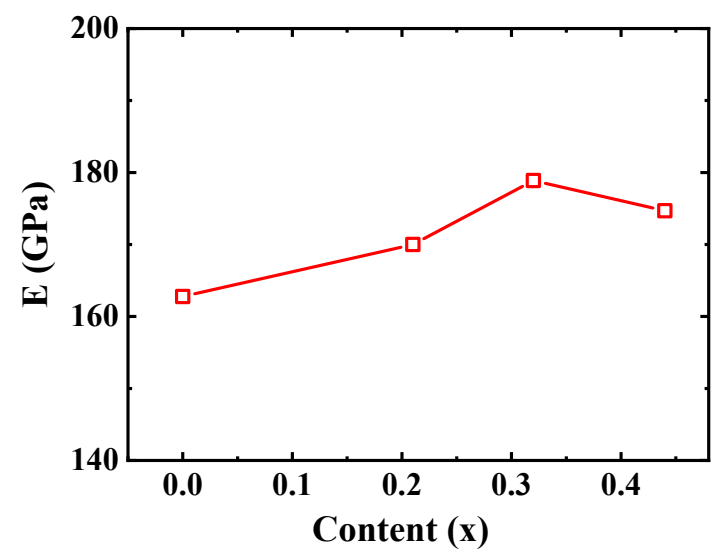

Figure $\mathrm{S3}$ Young's modulus for $\mathrm{Yb}_{0.3-\delta} \mathrm{Co}_{4} \mathrm{Sb}_{12} / \mathrm{xCoAl}$ samples.

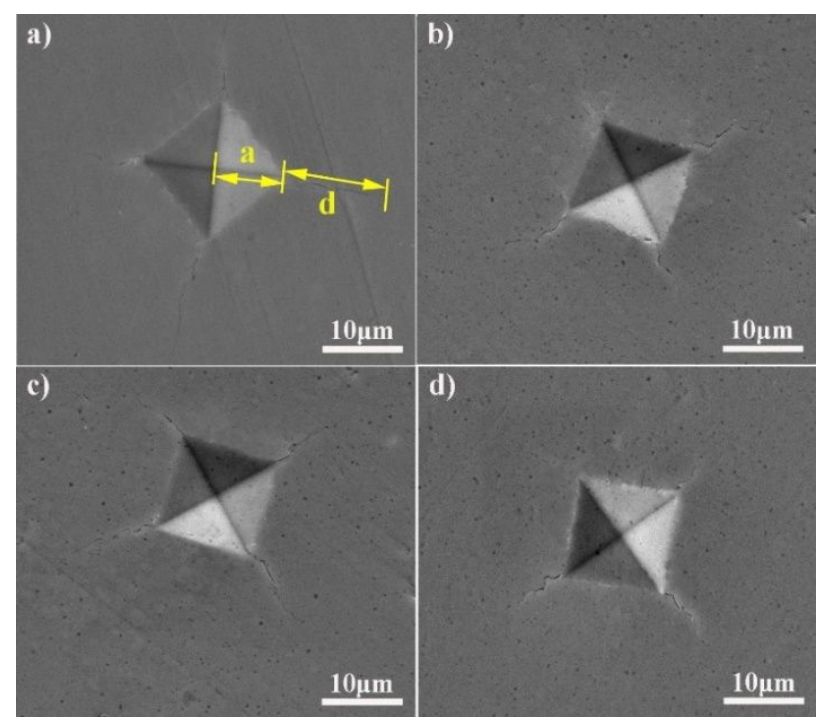

Figure S4 SEM images of Vickers-indentation cracks developed in samples. a) $\mathrm{Yb}_{0.29} \mathrm{Co}_{4} \mathrm{Sb}_{12}$, b) $\mathrm{Yb}_{0.22} \mathrm{Co}_{4} \mathrm{Sb}_{12} / 0.21 \mathrm{CoAl}$, c) $\mathrm{Yb}_{0.21} \mathrm{Co}_{4} \mathrm{Sb}_{12} / 0.32 \mathrm{CoAl}$, d) $\mathrm{Yb}_{0.18} \mathrm{Co}_{4} \mathrm{Sb}_{12} / 0.44 \mathrm{CoAl}$. 


\section{Reference}

[1] Ertl G. and Küppers J. Low Energy Electrons and Surface Chemistry, Weinheim: VCH, 1985; pp 6.

[2] Wood D. M. Classical size dependence of the work function of small metallic spheres. Phys. Rev. Lett. 1981, 46, 749-749. 